International Journal of Pure and Applied Mathematics

Volume 105 No. 4 2015, 805-809

ISSN: 1311-8080 (printed version); ISSN: 1314-3395 (on-line version)

url: http://www.ijpam.eu

doi: http://dx.doi.org/10.12732/ijpam.v105i4.19

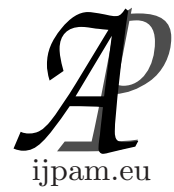

\title{
COMPACTNESS OF P-CLOSED SPACES VIA KATĚTOV'S METHOD AND ADHERENCE DOMINATORS
}

\author{
Terrence A. Edwards ${ }^{1}$, James E. Joseph ${ }^{2}$, \\ Myung H. Kwack ${ }^{3}$, Bhamini M.P. Nayar ${ }^{4} \S$ \\ ${ }^{1}$ Department of Mathematics \\ University of the District of Columbia \\ Washington, DC. 20008, USA \\ ${ }^{2,3}$ Emeritus, Department of Mathematics \\ Howard University \\ Washington, DC 20059, USA \\ 235 E Street NW \#709 \\ Washington, DC 20001, USA \\ ${ }^{3} 782$ Tiffany $\mathrm{Pl}$. \\ Concord, CA 94518, USA \\ ${ }^{4}$ Department of Mathematics \\ Morgan State University \\ Baltimore, MD 21251, USA
}

\begin{abstract}
An adherence dominator on a topological space $X$ is a function $\pi$ from the collection of filter bases on $X$ to the family of closed subsets of $X$ satisfying $\mathcal{A}(\Omega) \subset \pi(\Omega)$ where $\mathcal{A}(\Omega)$ is the adherence of $\Omega[10]$ and $\pi \Omega=$ $\bigcap_{\Omega} \pi F=\bigcap_{\mathrm{o}} \pi V$, where $\mathcal{O}$ represents the open members of $\Omega$. The notations $\pi \Omega$ and $\mathcal{A} \Omega$ are used for the values of the functions $\pi$ and $\mathcal{A}$. The $\pi$-adherence may be adherence $\mathcal{A}, \theta$-adherence [16], $u$-adherence [4], [5], [8], $s$-adherence [7], [9], $f$-adherence [6], $\delta$-adherence [14], etc., of a filter base. The theorems in [2], [3] and [12] on Hausdorff-closed, Urysohn-closed, and regular-closed spaces are subsumed in this paper as well as compactness of other p-closed spaces, using Katětov's method and adherence dominators.
\end{abstract}

AMS Subject Classification: 54D25, 54A05, 54A20

Key Words: filters, adherence dominator, compact, p-closed, Katětov

Received: October 10, 2015

(c) 2015 Academic Publications, Ltd. url: www.acadpubl.eu

$\S_{\text {Correspondence author }}$ 


\section{Introduction}

An adherence dominator (A.D.) on a topological space $X$ is a function $\pi$ from the collection of filter bases on $X$ to the family of closed subsets of $X$ satisfying $\Omega_{1} \subset \Omega_{2} \Rightarrow \pi \Omega_{2} \subset \pi \Omega_{1}$, and $\mathcal{A} \Omega \subset \pi \Omega$ where $\mathcal{A} \Omega$ is the adherence of $\Omega$ [10]. In [10] the concept of an adherence dominator was used to provide a frame work under which many characterizations of p-closed spaces and minimal p spaces were subsumed for $\mathrm{p}=$ Hausdorff, Urysohn, regular. This idea was also used to provide several new characterizations of such spaces as well as characterizations of compact spaces. In this article we present generalizations of the affirmative answers to questions raised in [1] and solved in [2], [3] and [12]. In [1] the following questions were stated as open problems: Is a Urysohn (regular) space in which every closed subset is Urysohn-closed (regular-closed) compact? The Hausdorff question was answered in the affirmative by Stone [15] using Boolean Rings and by Katětov [13] using topological methods. A new proof of this, using ultrafilters, was given in [11]. All of these questions were answered in the affirmative in [12] and more generalizations of these were given in [2] and [3]. Generalizations of theorems in [2] and [3], and others are given in this article, using the approach of Katětov, and the concept of adherence dominator.

\section{Preliminaries}

The following theorem comes from Zorn's Lemma.

Theorem 1. Let $\mathcal{U}$ be an ultrafilter on a space $X$ and $\mathcal{O}=\{O$ open in $X$ : $F \subset O$ for some $F \in \mathcal{U}\}$. Then $\mathcal{O}$ is an open ultrafilter on $X$, and $\pi \mathcal{U}=$ $\pi \mathcal{O}, \operatorname{adh} \pi \mathcal{O}=\{x\}$, for some $x \in X$.

Proof. Order the collection of $\mathcal{O}$ by inclusion and let $\mathcal{D}$ be the union of the elements of this collection. Then $\mathcal{D}$ is an upper bound for this collection. So, by Zorn, the proof is complete. To show that $\pi \mathcal{U}=\pi \mathcal{O}$ clearly, $\pi \mathcal{U} \subset \pi \mathcal{O}$. For the reverse inclusion, if $x \notin \pi \mathcal{U}$, there is an open set $V \in \mathcal{U}$ such that $x \in X-\pi V$. Thus, $x \notin \pi \mathcal{O}$. Hence $\pi \mathcal{U}=\pi \mathcal{O}$. To see that $a d h \pi \mathcal{O}=\{x\}$, for some $x \in X$, note that $\mathcal{O}$ is also an open ultrafilter, so $\operatorname{adh} \pi \mathcal{O}=\{x\}$, 


\section{Main Results}

Theorem 2. Let $\pi$ be an A.D. on a space $X$. Then $X$ is compact if and only if each closed subset is $\pi$-closed.

Proof. Clearly, every compact subset of a space is $\pi$-closed. Now, let $\mathcal{U}$ be an ultrafilter on $X$. Then by the theorem above $a d h \pi \mathcal{U}=\{x\}$ for some $x \in X$. Let $V \in \sum\{x\}$, the collection of open sets containing $x$. Then $F \cap(X-V)=\emptyset$ for some $F \in \mathcal{U}$, since otherwise $V, X-V \in \mathcal{U}$. Thus $\mathcal{U} \rightarrow x$.

Corollary 1. The following are equivalent for a Hausdorff (Urysohn) [regular] space $X$ :

1. $X$ is compact;

2. Each closed subset of $X$ is Hausdorff-closed (Urysohn-closed) [regularclosed] [2];

3. Each closed subset of $X$ is quasi Hausdorff-closed (quasi Urysohn-closed) [quasi regular-closed] [2];

4. Each closed subset of $X$ is $\theta$-closed (u-closed) [s-closed] [3].

The following corollary is left to the reader.

Corollary 2. The following are equivalent for a completely Hausdorff (completely regular) space $X$ :

1. $X$ is compact;

2. Each closed subset of $X$ is completely Hausdorff-closed (completely regularclosed);

3. Each closed subset of $X$ is quasi completely Hausdorff closed (quasi completely regular-closed). 


\section{References}

[1] M. P. Berri, J. R. Porter and R. M. Stephenson, Jr, A survey of minimal topological spaces, General Topology and Its Relations To Modern Analysis and Algebra,Proc. Conf. Kanpur (1968) (Academia Prague (1971), 93-114.

[2] T. A. Edwards, J. E Joseph, M. H. Kwack and B. M. P. Nayar, Compact spaces via p-closed subsets, Journal of Advanced studies in Topology, vol. 5 (2) (2014), 8-12.

[3] T. A. Edwards, J. E Joseph, M. H. Kwack and B. M. P. Nayar, Compactness via $\theta$-closed and $\theta$-rigid subsets, Journal of Advanced studies in Topology, vol. 5 (3) (2014), 28-34.

[4] M. S. Espelie, J. E. Joseph and M. H. Kwack, Applications of the u-closure operator, Proc. Amer. Math. Soc. 83 (1981), 167-174.

[5] L. L. Herrington, Characterizations of Urysohn-closed spaces, Proc. Amer. Math. Soc. 55 (1976), 435-439.

[6] L. L. Herrington, Characterizations of ompletely Hausdorff-closed spaces, Proc. Amer. Math. Soc. 55 (1976), 140-144.

[7] L. L. Herrington, Characterizations of regular-closed spaces, Math. Chronicle 9 (1977), 168-178.

[8] J. E. Joseph, Urysohn-closed and minimal Urysoh spaces, Proc. Amer. Math. Soc. 68 (2) (1978), 336-342.

[9] J. E. Joseph, Regular-closed and minimal regular spaces, Canad. Math. Bull. Vol. 22 (4), (1979),491 -497.

[10] J. E. Joseph, P-closed and minimal-P spaces from adherence dominators and graphs, Rev. Roumaine Math. Pures Appl. Vol. 25 No.7 (1980), 10471057.

[11] J. E. Joseph and B. M. P. Nayar, A new proof for a theorem of Stone and Katětov, International Journal of Pure and Applied Mathematics, Vol. 89, No.2, (2013) 287-288.

[12] J. E. Joseph and B. M. P. Nayar, A Hausdorff (Urysohn) [regular] space in which all of its closed sets are Hausdorff-closed (Urysohn-closed) [regularclosed] is compact, Journal of Advanced studies in Topology, vol. 5 (1) (2014), 6-8. 
[13] M. Katětov, Uber H-abgeschlossene und bikompakte raume, Casopis Pest. Mat.69 (1940), 36-49, (German).

[14] M. K. Singal and A. Mathur, A On nearly compact spaces, Boll. Un. Mat. Ital. (4) 2 (1969), 702-710,

[15] M. H. Stone, Applications of the theory of Boolean Rings to General Topology, Trans. Amer. Math. Soc. 41 No. 3 (1937), 375-481.

[16] N. V. Veličko, H-closed Topological Spaces, Mat. Sb. Vol. 70 (112) (1966), 98 - 112: Amer. Math. Soc. Transl. Vol. (2) 78 (1968), 103-118. 
